\title{
Information capacity of 3-D holographic data storage
}

\author{
D. BRADY \\ Department of Electrical and Computer Engineering, University of Illinois at \\ Urbana-Champaign, Urbana, Illinois, USA \\ D. PSALTIS \\ Department of Electrical Engineering, California Institute of Technology, \\ Pasadena, California, USA
}

Received 1 February; accepted 5 March 1993

Capacities for angular and wavelength multiplexed holographic data storage systems are considered. Limitations due to the spatial resolution of the recording and reconstructing fields and to the holographic recording process are derived.

\section{Introduction}

Photosensitive volume media have long been considered for applications in data storage. Proposals for volume holographic storage were developed shortly after the introduction of the laser and interest has continued at varying degrees of intensity since [1,2]. Despite this history, volume storage has not been incorporated into commercial systems. Optical data storage currently finds commercial viability only in low-cost data distribution on CD ROM and in quasi-archival storage on removable disk read-write drives. Both of these technologies were made possible by the development in the past twenty years of low-cost diode lasers.

Explanations of the failure of volume storage to achieve commercial success include materials oriented and fundamental limitations on recording on 3-D holograms [3, 4], noise issues in the reconstruction of holograms $[5,6]$, the lack of overlap between the wavelength range of inexpensive recording sources and the wavelength range of good holographic materials, the expense of mechanical and electrooptic equipment for scanning volume media, the lack of high-resolution/high-contrast 2-D spatial light modulators, the sophistication of the control cquipment required to form many grating holograms, and perhaps even insufficient cost-utility value for very large memories in currently available digital equipment. In our opinion, materials, source and modulator limitations are the most significant barriers. The importance of the modulator and control system is emphasized by the fact that until recently relatively few studies had even attempted many-hologram storage in volumc media [7-9], despite the often discussed potential, because hardware for automating the process was unavailable. Even now, the resolution and contrast of common liquid crystal modulators is inadequate for real-time data storage. 
Progress in source, modulator and materials research offers hope for alleviating barriers to volume data storage systems. Source technologies of potential importance include visible solid state sources, high-power diodes and broad temporal bandwidth solid state sources. While most experimental studies of volume storage have relied on gas lasers, inexpensive solid state sources are necessary for commercial applications. Gas lasers have been necessary to obtain power densities and wavelengths compatible with available photosensitive media. In the last decade, however, the power, coherence and wavelength range of solid state sources has improved dramatically. 2-D modulator technologies have also improved. 3-D data storage uses 2-D modulators to encode information for recording into the volume. If 3-D storage is to be competitive with 2-D data storage, the spatial resolution of these modulators must be comparable to the spatial resolution of 2-D storage systems. Ironically, this constraint makes optical disk technology the best candidate for volume control systems. Materials development has not been as dynamic as source and modulator work, mostly because materials studies must be specific to the data storage problem. A great deal of attention has been paid to photorefractive media, which, despite progress on spectral sensitivity and fixing, have not provided a full answer to the materials problem. More recently, organic media have begun to be investigated more closely.

Given recent improvements in source and modulator technologies and the availability of microprocessor-controlled recording systems, systematic experimental studies of volume storage are much more practical now than in the past. Despite the thirty-year history of volume holographic storage proposals, optimal designs for these systems are not well known and the information-theoretic principles behind their design have not been systematically presented. Our aim in this paper is to elucidate these principles. We consider both angularly and wavelength multiplexed multiple hologram storage. Both approaches are shown to support substantial information densities. The information capacity of a volume data storage system is determined by the space-bandwidth product, which is basically the spatial extent of the system divided by the resolution of the data-encoding mechanism. In optical storage, resolution is fundamentally limited by the optical wavelength. In practice, geometrical factors in system design further limit resolution. In the second section of this paper, we calculate the volume of Fourier space spanned by the control fields for various volume recording geometries. We discuss the capacity of these systems and the relative advantages of each approach.

The information capacity of volume holographic systems is strongly influenced by the information recording process. If data is stored nonholographically, for example by layered photolithographic construction of the volume, the space-bandwidth product of the probe system is the true limit of the information capacity. In holographically recorded systems, however, the most fundamental limitation on information capacity is the loss of dynamic range arising from multiple-exposure holography $[3,10]$. The diffraction efficiency of each hologram in a multiply exposed volume falls inversely with the square of the number of holograms recorded. In Section 3 we show that this problem limits the factor by which the capacity of a volume memory may exceed the capacity of a planar memory.

\section{The band volume}

The information capacity of 3-D data storage is fundamentally limited by the spatial resolution of the recording and reconstructing fields. One expects the information capacity to 
be approximately the holographic volume divided by the volume of the smallest feature, or voxel. A more formal estimate of the information capacity is obtained from Fourier analysis. A hologram is a dielectric modulation in 3-D. Assume that the modulation is nonzero only within the finite volume $V$ and that the 3-D spatial Fourier spectrum of the modulation occupies an approximate Fourier volume $\Omega$. We call $\Omega$ the band volume. If the modulation is sampled at points on a periodic lattice, the actual hologram might be recoverable if the volume of the unit cell of the reciprocal lattice exceeds $\Omega$. If $v$ is the volume of the unit cell of the sampling lattice, the volume of the unit cell on the reciprocal lattice is $1 / v$. The number of samples taken is $V / v$. The original hologram might be recovered if

$$
\Omega>1 / v
$$

An upper bound on the number of degrees of freedom stored in the hologram is therefore,

$$
S \leq V \Omega
$$

In principle, any grating with spatial frequency less than $2 / \lambda$ can be recorded and reconstructed using light of wavelength $\lambda$. This means that in monochromatic holographic system,

$$
\Omega \leq 32 \pi / 3 \lambda^{3}
$$

In a polychromatic system,

$$
\Omega \leq 32 \pi / 3 \lambda_{\min }^{3}
$$

where $\lambda_{\min }$ is the minimum wavelength used in the system [11]. In practice, it is both difficult and, as we argue in the next section, unwise to fully utilize the available band volume. In practical systems the spatial bandwidth covered by the recorded hologram is limited by the apertures of the control optics and the angular or wavelength tuning characteristics of the optical fields. In this section we evaluate the addressable band volume in angular and wavelength multiplexed holographic systems. In Section 3, we consider the implications of data encoding, reconstruction and recording algorithms on this limit.

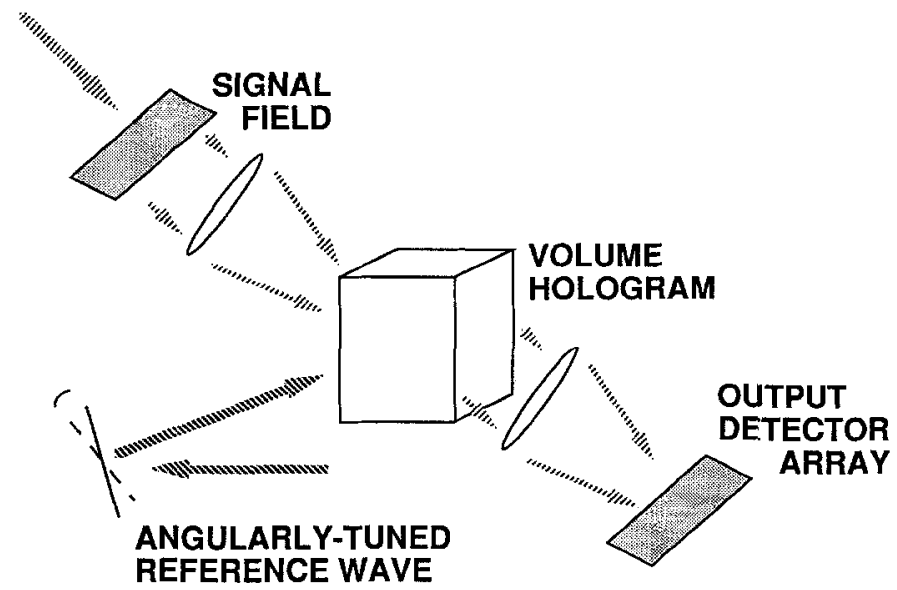

Figure 1 Angularly multiplexed hologram recording geometry. 
A 3-D holographic data storage system consists of a high-resolution 2-D spatial light modulator, such as film or optical disks, a reference field, a holographic volume and a sensor array. Data is encoded in 2-D and stored in 3-D by sequential recording of different 2-D fields. Between exposures the system geometry or recording wavelength is changed to address a new segment of the band volume. In this section we evaluate the band volume addressed by three types of recording systems. We first consider conventional angle multiplexed memories, which use a plane wave reference to record a page of data and scan from one page to the next by varying the angle of incidence of the reference field [12]. Second, we consider the band voiume addressed in a stationary plane wave reference system in which the band volume addressed in each exposure is changed by rotating the holographic volume. Third, we consider a stationary plane reference system in which the band volume addressed in each exposure is changed by changing the optical wavelength. We do not consider non-plane wave reference fields, which may be useful for data encoding but which do not substantially affect the band volume of the system.

An angularly multiplexed holographic storage system is sketched in Fig. 1. Signals generated on a spatial light modulator (SLM) interfere in a photosensitive volume with light from an angularly tuned reference source to create a spatially modulated index of refraction. The wave interactions of this system can equivalently be sketched in Fourier space, as shown in Fig. 2. The spherical shell is the wave normal surface at the recording wavelength. The wave vectors representing the signals form a cone and the wavevectors of the reference fields are represented by an arc. In considering Fourier space, we make a distinction between the optical Fourier space sketched in Fig. 2 and 'grating space'. Grating space is the Fourier transform space of the holographic modulation. In Fig. 2, the range of grating frequencies addressed is the range of difference vectors between the wavevectors of the signal field and the reference field. Let $\theta_{0}$ represent the angle between the centre axes of the signal field and the reference field. The angle between a particular wavevector in the signal (reference) field and the $z$ axis is $\theta_{\mathrm{S}}\left(\theta_{\mathrm{r}}\right)$. Reference fields lie entirely in the $z-x$ plane. The angle between the projection of a signal field in the $x-y$ plane and the $x$ axis is $\phi_{\mathrm{s}}$. We

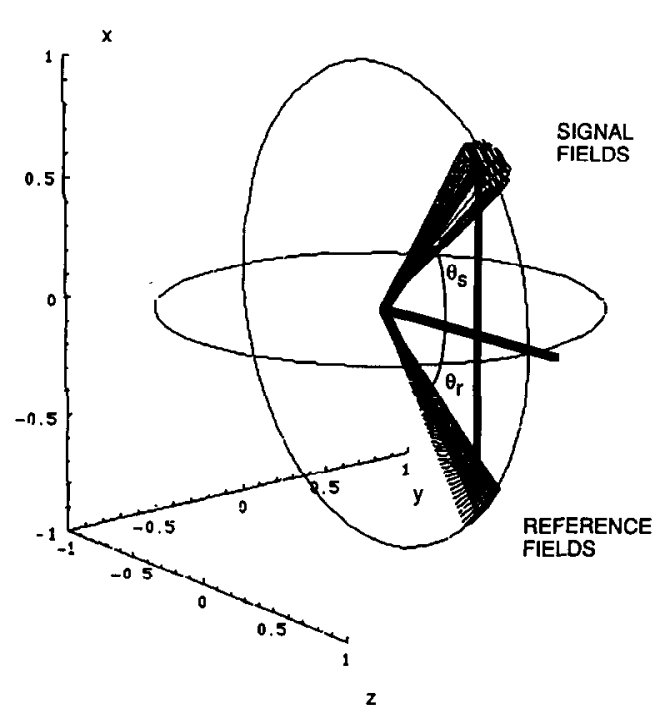

Figure 2 Fourier space representation of angularly multiplexed recording fields. 


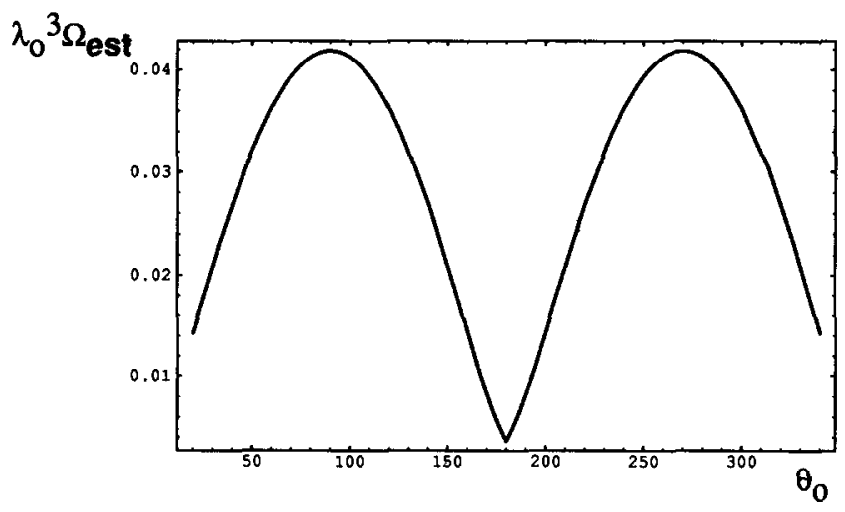

Figure $3 \Omega_{\text {est }}$ as a function of $\theta_{0}$ for $\Delta \theta=10^{\circ}$.

assume that the signal fields occupy a circular region on the normal sphere of angular radius $\Delta \theta$. The wavevectors in the signal field are

$$
\boldsymbol{k}_{\mathrm{s}}=k_{0} \sin \theta_{\mathrm{s}} \cos \phi_{\mathrm{s}} \hat{x}+k_{0} \sin \theta_{\mathrm{s}} \sin \phi_{\mathrm{s}} \hat{y}+\cos \theta_{\mathrm{s}} \hat{z}
$$

where $-\Delta \theta<\theta_{\mathrm{s}}-\theta_{0} / 2<\Delta \theta$,

$$
-\cos ^{-1}\left[\frac{\cos \Delta \theta-\cos \left(\theta_{0} / 2\right) \cos \theta_{\mathrm{s}}}{\sin \left(\theta_{0} / 2\right) \sin \theta_{\mathrm{s}}}\right]<\phi_{\mathrm{s}}<\cos ^{-1}\left[\frac{\cos \Delta \theta-\cos \left(\theta_{0} / 2\right) \cos \theta_{\mathrm{s}}}{\sin \left(\theta_{0} / 2\right) \sin \theta_{\mathrm{s}}}\right]
$$

and $\Delta \theta<\theta_{0} / 2<P i / 2-\Delta \theta$. The wavevectors in the reference field are

$$
\boldsymbol{k}_{\mathrm{s}}=-k_{0} \sin \theta_{\mathrm{r}} \hat{x}+\cos \theta_{\mathrm{r}} \hat{z}
$$

where $-\Delta \theta<\theta_{\mathrm{r}}-\theta_{0} / 2<\Delta \theta$. The grating wavevectors between the reference and signal fields are

$$
\boldsymbol{K}_{\mathrm{g}}=-\boldsymbol{k}_{\mathrm{s}}-\boldsymbol{k}_{\mathrm{r}}
$$

An upper bound of the volume in grating space spanned by the recording fields is obtained by multiplying the range of $\boldsymbol{K}_{\mathrm{g}}$ along the three Cartesian coordinate axes

$$
\begin{aligned}
\Omega_{\mathrm{est}} & =\left(1 / 8 \pi^{3}\right)\left(\operatorname{Max}\left(K_{\mathrm{gx}}\right)-\operatorname{Min}\left(K_{\mathrm{g} x}\right)\right)\left(\operatorname{Max}\left(K_{\mathrm{g} y}\right)-\operatorname{Min}\left(K_{\mathrm{g} y}\right)\right)\left(\operatorname{Max}\left(K_{\mathrm{g} z}\right)-\operatorname{Min}\left(K_{\mathrm{g} z}\right)\right) \\
& =\frac{8}{\lambda_{0}^{3}} \sin ^{2} \Delta \theta \sin \frac{\theta_{0}}{2} \times \begin{cases}2 \cos \left(\theta_{0} / 2\right) \sin \Delta \theta & \text { for }\left|\pi / 2-\theta_{0} / 2\right|>\Delta \theta \\
1-\sin \left(\theta_{0} / 2-\Delta \theta\right) & \text { for } \pi / 2-\Delta \theta<\theta_{0} / 2<\pi / 2 \\
1-\sin \left(\theta_{0} / 2+\Delta \theta\right) & \text { for } \pi / 2+\Delta \theta>\theta_{0} / 2>\pi / 2\end{cases}
\end{aligned}
$$

In Fig. $3 \lambda_{0}^{3} \Omega_{\text {est }}$ is plotted as a function of $\theta_{0}$. We assume that $\Delta \theta=10^{\circ}$. The band volume of the system at $\theta_{0}=90^{\circ}$ is 11.4 times the minimum band volume, which occurs at $\theta_{0}=180^{\circ}$. The difference in capacity between the transmission and reflection geometries is apparent when $\Delta \theta \ll 1$, in which case

$$
\Omega_{\mathrm{est}}\left(\theta_{0}=90^{\circ}\right) \approx 16 \Delta \theta^{3} / \lambda_{0}^{3}
$$

and

$$
\Omega_{\mathrm{est}}\left(\theta_{0}=180^{\circ}\right) \approx 4 \Delta \theta^{4} / \lambda_{0}^{3}
$$




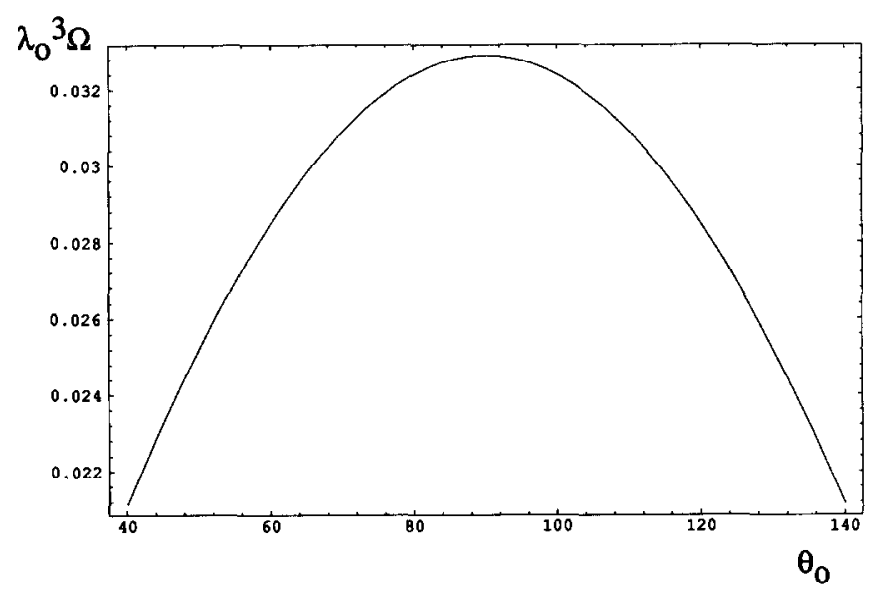

Figure $4 \lambda_{0}^{3} \Omega$ as a function of $\theta_{0}$ for $\Delta \theta=10^{\circ}$.

A more exact analysis of the band volume is based on the differential volume

$$
\begin{aligned}
\mathrm{d} v & =\frac{1}{8 \pi^{3}}\left|\frac{\partial \boldsymbol{K}_{\mathrm{g}}}{\partial \theta_{\mathrm{r}}}\left[\frac{\partial \boldsymbol{K}_{\mathrm{g}}}{\partial \theta_{\mathrm{s}}} \times \frac{\partial \boldsymbol{K}_{\mathrm{g}}}{\partial \phi_{\mathrm{s}}}\right]\right| \mathrm{d} \phi_{\mathrm{s}} \mathrm{d} \theta_{\mathrm{s}} \mathrm{d} \theta_{\mathrm{r}} \\
& =\frac{1}{\lambda_{0}^{3}}\left|\cos \theta_{\mathrm{r}} \sin ^{2} \theta_{\mathrm{s}} \cos \phi_{\mathrm{s}}+\frac{1}{2} \sin \theta_{\mathrm{r}} \sin 2 \theta_{\mathrm{s}}\right| \mathrm{d} \phi_{\mathrm{s}} \mathrm{d} \theta_{\mathrm{s}} \mathrm{d} \theta_{\mathrm{r}}
\end{aligned}
$$

The differential volume is integrated numerically over the range of the signal and reference fields to find the band volume of the recording system. As shown in Fig. 4, the actual band volume is somewhat less than the estimate of Fig. 3. The band volume at $\theta_{0}=90^{\circ}$ is plotted as a function of $\Delta \theta$ in Fig. 5. As shown in the figure, a large numerical aperture allows a sizeable fraction of the total band volume to be addressed in the right-angle transmission geometry.

Important differences in the band volume are obtained by rotating the holographic medium instead of the reference. Qualitatively, rotation of the medium may be preferred because the angle between the optical axes of the reference and signal fields remain at the optimal value as the sample is rotated. Since rotation of the sample leads to no shift in

$$
\lambda_{0}^{3} \Omega
$$

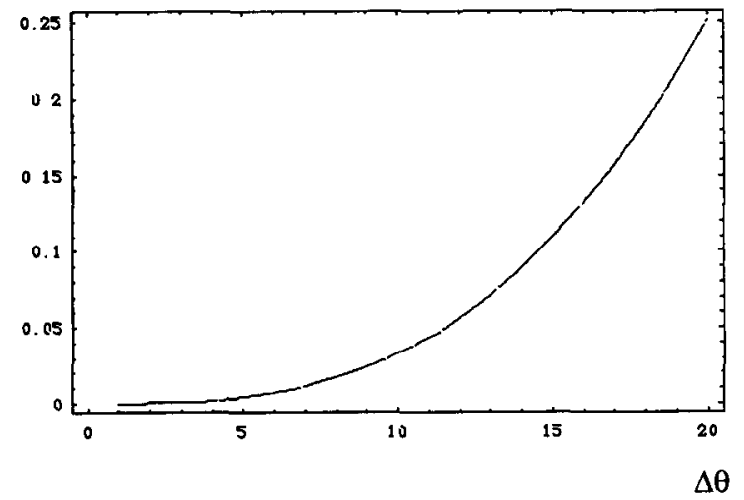

Figure $5 \lambda_{0}^{3} \Omega$ as a function of $\Delta \theta$ for $\theta_{0}=90^{\circ}$. 


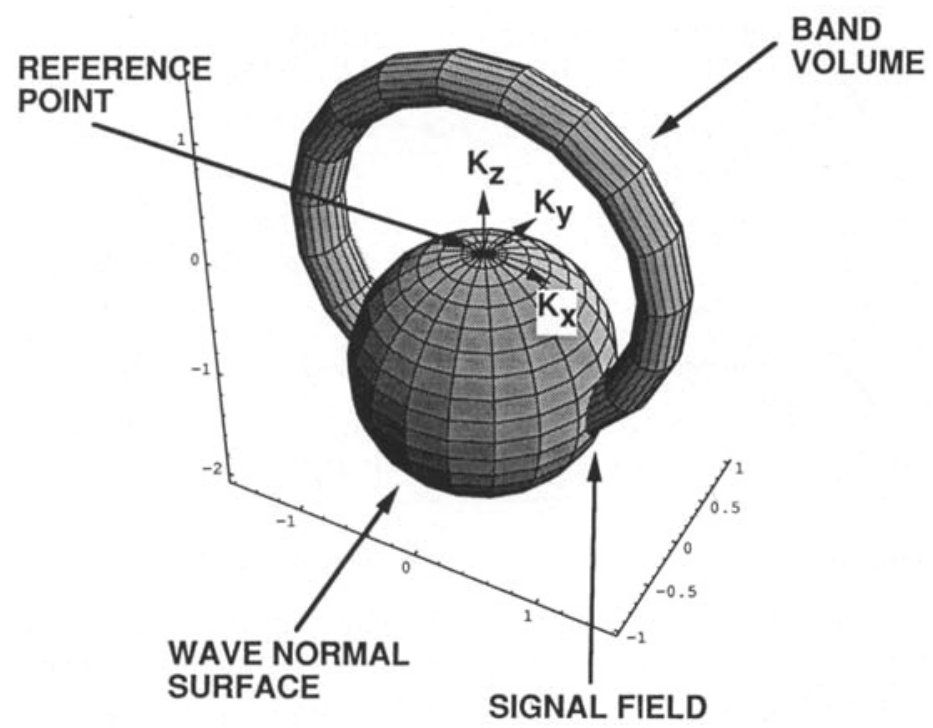

Figure 6 Band volume for a rotating medium system. The length scale for the three axes is $k_{0}$. The intersection of the toroidal band volume with the wave normal sphere is the circularly symmetric signal field In this figure, the reference and signal fields are orthogonally propagating and the half-angular width of the signal field is $10^{\circ}$.

beam overlap or relative propagation direction, the angular range of this rotation is likely to be much larger than the range of the reference angle. The grating wavevectors recorded between a plane wave reference and a circular signal field correspond to the vectors between one wavevector in the reference field of Fig. 2 and all points in the signal field. We can consider the intersection point of the reference wavevector and the wave normal surface to be the origin of the grating space. Rotating the recording medium results in an identical rotation in grating space. The band volume addressed by rotating the volume is the volume covered by rotating the Fourier representation of the signal field about the intersection of the reference wavevector with the wave normal sphere. This volume, sketched in Fig. 6, is a toroid. The sphere in Fig. 6 is a wave normal surface

$$
\lambda_{0}{ }^{3} \Omega
$$

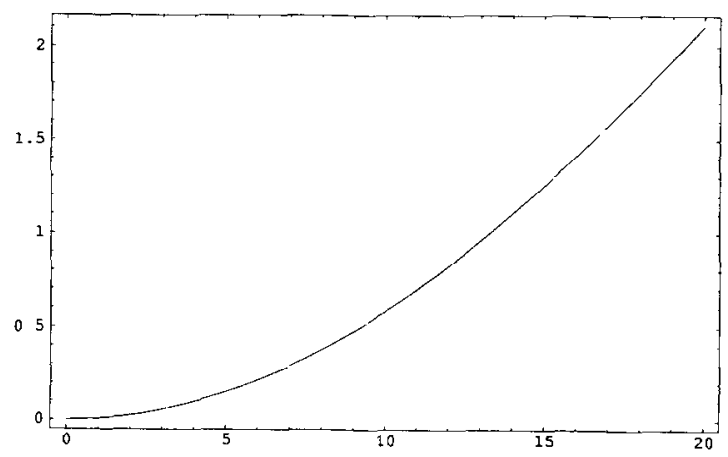

Figure 7 Effective band volume for a rotating sample as a function of $\Delta \theta$. 


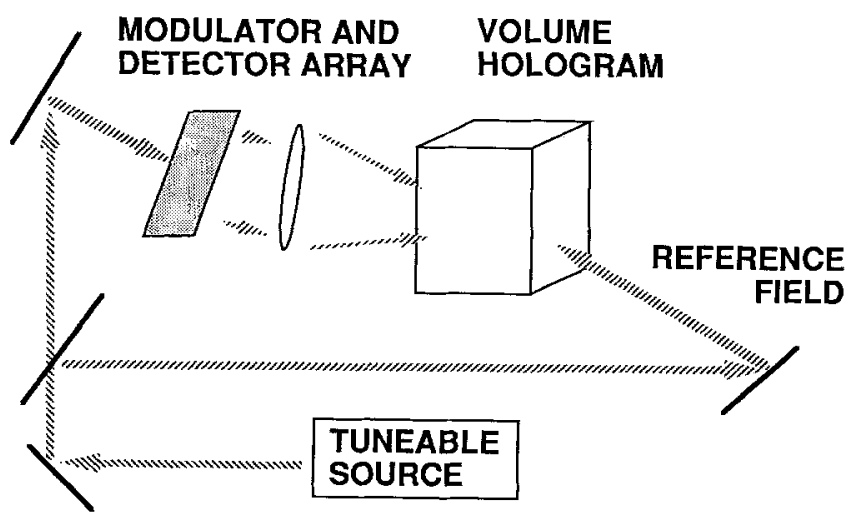

Figure 8 Recording geometry for wavelength multiplexed volume data storage.

for the recording fields. The total potential band volume for recording at this wavelength is a sphere of twice the radius of the sphere shown centred on the reference point. The actual band volume accessible to the recording fields is the toroid. To account for inversion symmetry in grating space, the effective band volume for this system must be taken to be half the volume of the toroid. The band volume can be shown to be

$$
\lambda_{0}^{3} \Omega=\left(\pi^{2} / 2\right) \sin \theta_{0}(1+\cos \Delta \theta)\left(1-\cos ^{4} \Delta \theta\right)
$$

where $\theta_{0}$ and $\Delta \theta$ are the reference and signal axis angles and signal angular width defined above. The band volume at $\theta_{0}=90^{\circ}$ is plotted as a function of $\Delta \theta$ in Fig. 7. If we limit the rotational range of the recording medium to less than $180^{\circ}$, the effective band volume is proportionally decreased.

Wavelength multiplexed holograms are an alternative to angularly multiplexed holograms [13]. The basic geometry for a WDM storage system is sketched in Fig. 8. A tunable source is used to record a series of holograms at different wavelengths. We do not consider simultaneous angular and wavelength tuning because the optimal signal field geometries for the two cases are orthogonal. A cross-section of the band volume that can

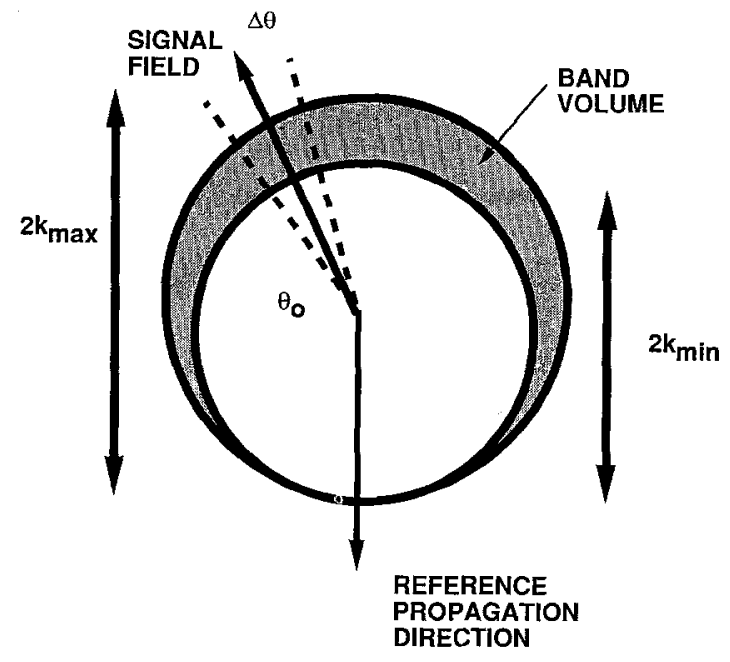

Figure 9 Cross-section of the band volume of wavelength multiplexed fields. The full band volume is the cross-section rotated about the vertical axis. 
$\lambda_{0}{ }^{3} \Omega$

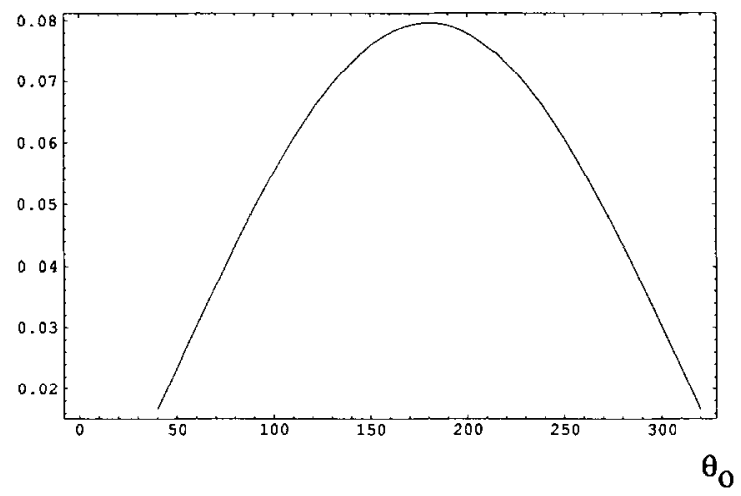

Figure $10 \lambda^{3} \Omega$ as a function of $\theta_{0}$ for the wavelength multiplexed case. In this case $\Delta \lambda=0.1 \lambda$ and $\Delta \theta=10^{\circ}$.

be addressed by reference fields propagating along a fixed direction is shown in Fig. 9. The reference fields propagate straight down and, as above, are separated from the optical axis of the signal fields by an angle $\theta_{0}$. The signal fields cover a circularly symmetric solid angle of radius $\Delta \theta$. One can see from Fig. 9 that the reflection geometry is optimal for wavelength multiplexed holograms because the band volume is thickest at the top. The differential band volume for the WDM case is

$$
\begin{aligned}
\mathrm{d} v & =\frac{1}{8 \pi^{3}}\left|\frac{\partial \boldsymbol{K}_{\mathrm{g}}}{\partial \lambda}\left[\frac{\partial \boldsymbol{K}_{\mathrm{g}}}{\partial \theta_{\mathrm{s}}} \times \frac{\partial \boldsymbol{K}_{\mathrm{g}}}{\partial \phi_{\mathrm{s}}}\right]\right| \mathrm{d} \phi_{\mathrm{s}} \mathrm{d} \theta_{\mathrm{s}} \mathrm{d} \lambda \\
& =\frac{1}{\lambda^{4}}\left|\sin \theta_{\mathrm{s}}+\sin \theta_{\mathrm{r}} \sin ^{2} \theta_{\mathrm{s}} \cos \phi_{\mathrm{s}}-\frac{1}{2} \cos \theta_{\mathrm{r}} \sin 2 \theta_{\mathrm{s}}\right| \mathrm{d} \phi_{\mathrm{s}} \mathrm{d} \theta_{\mathrm{s}} \mathrm{d} \lambda
\end{aligned}
$$

We find the bandwidth of the holographic storage system by integrating the differential band volume over the solid angle of the signal field and the spectral range of the source. Figure 10 shows the band volume as a function of $\theta_{0}$ for a $20^{\circ}$ signal field and a $10 \%$ spectral width. The maximum bandwidth for WDM holograms occurs in the reflection geometry. As shown in Fig. 11 , the bandwidth at $\theta_{0} / 2=90^{\circ}$ is approximately linear in

$\lambda_{0}{ }^{3} \Omega$

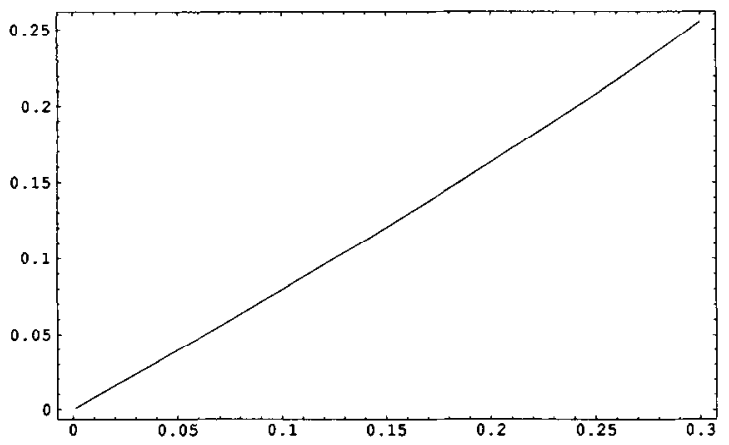

Figure $11 \lambda^{3} \Omega$ as a function of $\Delta \lambda$ for $\Delta \lambda \quad \Delta \theta=10^{\circ}$. 
the spectral width. A $5 \%$ spectral width, corresponding to $40 \mathrm{~nm}$ on an $800 \mathrm{~nm}$ centre wavelength, yields a capacity similar to the maximum bandwidth for the angularly multiplexed case at this numerical aperture.

To this point we have limited our discussion to strictly geometric factors limiting storage capacity. In practice it is important to consider a number of other factors in designing a volume data storage system. Refraction at material interfaces, polarization states and materials anisotropies, aperture sizes, scattering noise and crosstalk all impact system design. For example, we have seen that the $90^{\circ}$ propagation geometry yields optimal storage capacity for angularly multiplexed holograms. Brewster constraints prohibit polarization-preserving scattering of the polarization in the plane of the signal and reference optical axes in this geometry. In anisotropic media with strong scattering for the in-plane polarization, implementation of the orthogonally propagating geometry may be challenging.

Our analysis of information capacity as a function of the signal solid angle has been based on the value of the solid angle inside the holographic material. In practice, refraction at the holographic interface changes both the effective solid angle and the effective wavelength. If the boundary of the holographic material is required to be flat, refraction compresses the free-space solid angle of the control fields. For orthogonally diffracting angularly multiplexed holograms, compression of the solid angle and the wavelength offset each other if the reference and signal fields enter the holographic medium from orthogonal faces. The information capacity in this case can be shown to be approximately independent of the holographic medium's index of refraction. In reflection geometry WDM holograms, the principal grating wavevectors are normal to the interfaces. Since the reduction in the effective wavelength is maximal along this direction, the increased capacity due to shorter wavelengths dominates the compression of the signal solid angle and refraction can substantially increase storage capacity. Our analysis need not be limited to flat holographic interfaces, however. While curved interfaces may be difficult to achieve using crystalline materials, they can easily be fabricated for organic media. If we allow the hologram to have spherical interfaces, then refraction does not limit the solid angle of the signal field.

\section{Data storage and retrieval}

While the product of the spatial volume and band volume of a holographic system is a good estimate of the potential information capacity, this capacity may be difficult to achieve in practice. Two aspects of information storage and retrieval limit the storage capacity. Information storage is challenging as a result of the loss in dynamic range inherent in holographic control [3]. This problem limits the number of exposures that can be superimposed in a volume medium. Information retrieval is challenging because digital data storage requires that each bit stored must be independently extractable with low signal to noise. Since the impact of the control problem is easier to understand if we know the coding scheme, we consider the difficulties of discrete data retrieval first.

Suppose that a hologram that uses the full band volume has been recorded. The problem of retrieving the stored data is equivalent to the general problem of optical tomographic imaging, but in a data storage systcm onc is less likcly to use phase-sensitive detection techniques or to consider correlations between reconstruction views to extract data. Thus, we must show that the number of independent parameters that can easily be extracted from the scattered fields is comparable to the product $V \Omega$. We assume that 
the recorded hologram is a permittivity modulation described by

$$
\Delta \epsilon(\boldsymbol{r})=V(\boldsymbol{r}) \sum_{n} \alpha(\boldsymbol{n}) \mathrm{e}^{\mathrm{j} K n \cdot \boldsymbol{r}}
$$

where $\boldsymbol{n}$ is a vector on a three-dimensional number lattice and the sum is over grating wavevectors within the controlled band volume. $V(\boldsymbol{r})$ is a function describing the extent of the holographic volume, analogous to the pupil function of Fourier optics. $V(\boldsymbol{r})$ is 1 inside the controlled volume and 0 elsewhere. Since scattering from many exposure holograms is always weak, the Born approximation is appropriate to analysing the scattered field. The scattered field due to a plane wave probe with wavevector $\boldsymbol{k}_{\mathrm{r}}$ is

$$
\psi_{\mathrm{s}}(\boldsymbol{r})=\iiint_{V} \mathrm{~d}^{3} r^{\prime} G\left(\boldsymbol{r}-\boldsymbol{r}^{\prime}\right) \Delta \epsilon(\boldsymbol{r}) \mathrm{e}^{\mathrm{j}_{\mathrm{r}} \cdot \boldsymbol{r}}
$$

where $G\left(\boldsymbol{r}-\boldsymbol{r}^{\prime}\right)$ is a Green function. The three-dimensional Fourier transform of the scattered field is

$$
\Psi_{\mathrm{s}}(\boldsymbol{k})=\sum_{\boldsymbol{n}} \alpha(\boldsymbol{n}) \mathcal{V}\left(\boldsymbol{K} \boldsymbol{n}+\boldsymbol{k}_{\mathrm{r}}-\boldsymbol{k}\right) \mathcal{G}(\boldsymbol{k})
$$

where $\mathcal{V}(\boldsymbol{k})$ and $\mathcal{G}(\boldsymbol{k})$ are the Fourier transforms of $V(\boldsymbol{r})$ and $G(\boldsymbol{r})$.

If the probe wave is Bragg matched to the $n_{\mathrm{p}}$-th grating component in $\Delta \epsilon$ then a signal proportional to $\alpha\left(\boldsymbol{n}_{\mathrm{p}}\right)$ is observed in Fourier component of the scattered field at wavevector $\boldsymbol{k}=\boldsymbol{k}_{\mathrm{r}}+\boldsymbol{K} \boldsymbol{n}_{\mathrm{p}}$. If $\alpha\left(\boldsymbol{n}_{\mathrm{p}}\right)$ is our stored data bit, the signal-to-noise ratio for detection from the spatial spectrum is the ratio of the power in the Bragg matched term to the total power in the mismatched terms. Assuming that $\alpha(\boldsymbol{n})$ is a random variable with a uniform phase distribution, the SNR is

$$
\mathrm{SNR}=\frac{|\mathcal{V}(0)|^{2}}{\sum_{n \neq n_{\mathrm{p}}}\left|\mathcal{V}\left(K\left(n-n_{\mathrm{p}}\right)\right)\right|^{2}}
$$

Given $V(r)$, it is straightforward to calculate the SNR for various grating densities. For example, if the holographic volume is a sphere of radius $R$ then

$$
\mathcal{V}(\boldsymbol{k})=\frac{4 \pi}{k^{3}}(\sin k R-k R \cos k R)
$$

where $k=|\boldsymbol{k}|$. Figure 12 shows the SNR for a spherical volume as a function of the

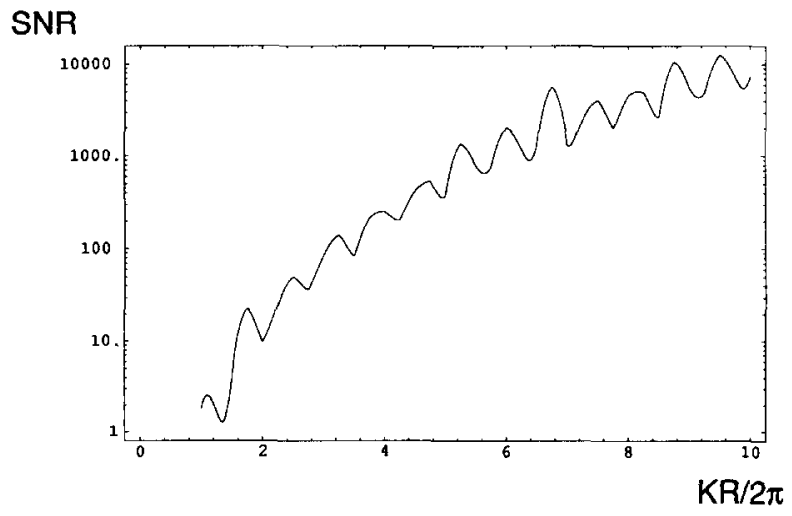

Figure 12 Ambiguity SNR of the amplitude of a single grating in a uniformly sampled band volume as a function of the sampling rate, $K$. The sampling lattice is face-centred cubic and the 9260 nearest neighbours to Bragg match are used to calculate the SNR. 


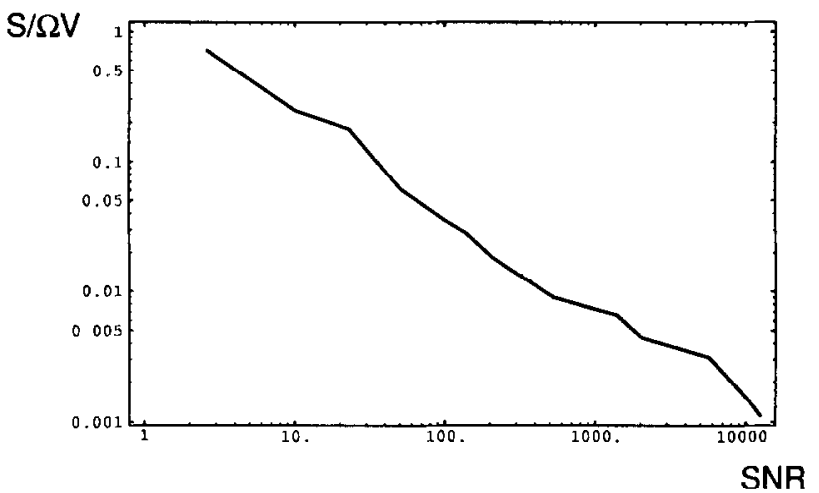

Figure 13 Information capacity $S$, in units of $V \Omega$, as a function of ambiguity SNR.

fundamental grating spacing in the band volume, $K$. The sampling lattice is a face-centredcubic on which $K$ represents the cubic lattice spacing. $K=2 \pi / R$ reuslts in an SNR of 1.8 and corresponds to an information capacity of

$$
S=4 \Omega /(K / 2 \pi)^{3}=3 V \Omega / \pi
$$

While this information capacity is comparable to the results of Section 2, one is likely in practice to demand greater SNR. $S / \Omega V$ is plotted as a function of SNR in Fig. 13.

As mentioned above, the number of exposures that can be made in a volume is fundamentally limited. The diffraction efficiency for each image in a series of exposures falls as the square of the number of exposures, $N$, and is independent of the number of degrees of freedom per stored image, $M$ [3]. The diffraction efficiency into a single component of a hologram therefore scales as $1 / N^{2} M$. Noting that $S=N M$, the diffraction efficiency of a single grating component of a multiple exposure hologram is

$$
\eta=\frac{\eta_{\max }}{N^{2} M}=\frac{\eta_{\max }}{S N}
$$

where $\eta_{\max }$ is the saturation diffraction efficiency of a single grating hologram in the photosensitive medium. If the photosensitivity of the material is such that very strong holograms may be recorded, the effective value of $\eta_{\max }$ may exceed 1 [10].

The inverse scaling of $\eta$ with $N$ has several implications. The most obvious is that it is better to record a lot of gratings in fewer exposures than a few gratings in a lot of exposures. To minimize $N$, one may choose not to fully populate the band volume with gratings. To record grating wavevectors on a cubic lattice in the band volume, one does

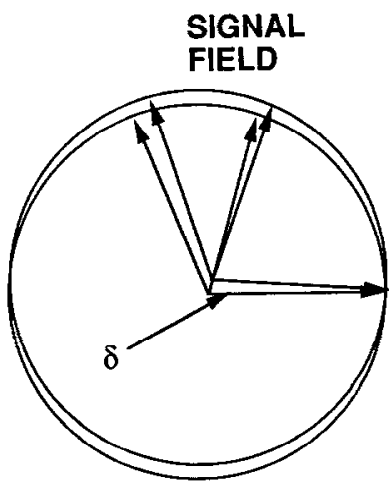

REFERENCE

FIELD

Figure 14 Displacement of the recording fields in grating space for a discrete rotation of holographic medium The displacement is maximal if the signal and reference fields are orthogonally propagating. 
not use the full space-bandwidth product of the recording field in each exposure. To record holograms of images that use the full space-bandwidth product of the recording system in each exposure, one rotates the hologram or detunes the wavelength by more than is required to record new gratings between the reference and signals near the axis of the signal field. Figure 14 shows the displacement of recording fields for a discrete rotation of the recording medium by $\delta$. To record $N$ exposures, we set $\delta=\pi / N$. Figure 14 shows that, as expected, the change in the gratings addressed is maximal for orthogonally propagating reference and signal fields. For a fixed rotation, the change in the grating recorded is larger near the centre of the signal field than near the edge of the signal field. For example, if $\Delta \theta=10^{\circ}$, the difference in the displacement from the original signal field to the rotated signal field between the centre of the signal field and the edge is $15 \%$. This means that less than $15 \%$ of the band volume must be wasted when the full spacebandwidth of the signal fields is used in every exposure. The loss of band volume due to this effect is somewhat less in WDM systems.

A more serious implication of the inverse scaling of $\eta$ with $N$ is the implied limitation on the capacity of the holographic volume. A good estimate of $\eta_{\max }$ is obtained by multiplying the reflectance in each grating period by the approximate number of fringes crossed by the probe field, which yields

$$
\eta_{\max }=\left(\frac{\Delta n}{n} \frac{n V}{\sigma \lambda}\right)^{2}
$$

where $\Delta n_{\max }$ is the maximum photoinduced change in refractive index and $\sigma$ is the crosssection of the holographic volume. Substituting in Equation 21 and solving for $S$ yields

$$
f=\frac{\lambda^{2} S}{\sigma}=\frac{\Delta n^{2}}{\eta} \frac{1}{N} \frac{V^{2}}{\sigma^{3}}
$$

$f$, the ratio of the number of bits stored in the volume and the number that might be stored on its surface, is a measure of the effectiveness of the holographic storage system. The maximum value of $f$ is $N$. Setting $f$ equal to $N$ in Equation 23 and solving for $N$, we find that the maximum value of $f$ is

$$
f_{\max }=N=\frac{\Delta n}{\sqrt{\eta}} \frac{V}{\sigma^{3 / 2}}
$$

For a spherical volume,

$$
f_{\max }=\frac{\Delta n}{\sqrt{\eta}} \frac{4}{3 \sqrt{\pi}}
$$

The minimum acceptable value of $\eta$ is key to evaluating $f$. If, for example, we wish to detect 1000 electrons in $30 \mathrm{~ms}$ for each stored bit using a detector of quantum efficiency 0.5 and $1 \mathrm{eV}$ photons, we require 10 femtowatts of optical power per data bit. If we read the hologram with a $10 \mathrm{~mW}$ source, this implies that

$$
\eta \geq 10^{-12}
$$

For a spherical volume with $\Delta n=10^{-3}$ this value of $\eta$ yields $f=750$. If, on the other hand, $\Delta n=10^{-5}, f$ is only 7.5. Of course, different values of $f$ are obtained with different sample shapes. For example, a long filament may have very small cross-section and large 
volume. However, increasing the ratio of $V / \sigma^{3 / 2}$ implies a decrease in $\Delta \theta$. In true volume storage one maximizes $\Delta \theta$, in which case $V / \sigma^{3 / 2}$ is of order 1 .

We have shown that the capacity of a holographically controlled volume memory exceeds the capacity of a surface memory of the same cross-section by a constant factor. This means that the capacity of the volume memory does not increase linearly with increasing volume. Of course, capacity increases linearly with volume if a new recording volume replaces the old. This means that even in holographic systems it is important to maintain as much locality as possible in data storage. Ideally, the storage system is designed with the minimum useful holographic volume and a mechanism for scanning the recording system across a larger volume.

\section{Conclusion}

System design and geometry dramatically impact the capacity of volume data storage systems. Given finite signal space-bandwidth product, we have derived optimal geometries for monochromatic and polychromatic storage. Neglecting recording difficulties, the data storage capacities of monochromatic and polychromatic holographic systems do not differ significantly. If data is holographically recorded, however, the useful holographic volume is severely limited. We have shown that the information capacity of a holographically controlled volume is only a constant factor greater than the surface capacity, which means that the capacity does not scale as the volume divided by the cubic wavelength. To avoid the loss in dynamic range resulting from grating superposition, one should minimize the size of the holographic volume recorded and displace new media into the recording volume between recording cycles. This displacement is likely to be easier in wavelength multiplexed systems.

\section{References}

1. P. J. VAN HEERDEN, Appl. Opt. 2 (1963) 393.

2. A. KOZMA and E. S. BARREKETTA (eds), Appl. Opt. 13(4) (1974); special issue on optical storage of digital data.

3. D. BRADY and D. PSALTIS, J. Opt. Soc. Am. A 9 (1992) 1167.

4. K. BLØTEKJAER, Appl. Opt. 18 (1979) 57.

5. W J BURKE and P. SHENG, J. Appl. Phys. 48 (1977) 681.

6. C. GU, J. HONG, I. MCMICHAEL, R. SAXENA and F. MOK, J. Opt. Soc. Am. A 9 (1992) 1978.

7. D. L. STAEBLER, W. J. BURKE, W. PHILlIPS and J. J. AMODEI, Appl. Phys. Lett. 26 (1975) 182.

8. R. A. BARTOLINI, A. BLOOM and J. S. ESCHER, Appl. Phys. Lett. 28 (1976) 506.

9. F MOK, M. TACKITT and H. M. STOLL, Opt. Lett. 16 (1991) 605.

10. J. HONG, P. YEH, D. PSALTIS and D. J. BRADY, Opt. Lett. 15 (1990) 344.

11. C. GU, Optical neural networks using volume holograms, $\mathrm{PhD}$ Thesis, California Institute of Technology (1990).

12. E N. LEITH, A KOZMA, J. UPATNIEKS, J. MARKS and N. MASSEY, Appl. Opt. 5 (1966) 1303.

13. G. A. RAKULJIC, V. LEYVA and A. YARIV, Opt. Lett. 17 (1992) 1471. 